\title{
MODIFY THE JOHANNES ITTEN CIRCLE OF COLORS TO CREATE HARMONIOUS COLOR GROUPS TO DEVELOP THE TEACHING OF COLOR SCIENCE
}

Muhammad Ali Mahmoud NASRA *

Faculty of Art Education, Helwan University, Egypt

\begin{abstract}
The current study falls within the scope of experimental research that is concerned with the concept of color harmony, COLOR HARMONY, as an investigator of one of the aesthetic phenomena in design, which aims to develop compatible color groups that contribute to the development of teaching color science studying plastic arts, especially in the field of design and its structural and aesthetic foundations. Where the researcher seeks, through a careful analytical reading of the color circle of the German scientist Johannes Atten-Johannes IT 1823-1749 (TEN AD), which is the most commonly used as a main component of the curricula for teaching color science and its characteristics in many international art academies, to make a modification in its design And its shape allows the creation of compatible color groups that are in total multiples of what it allows through its current design, and based on the system that Athen adopted in reaching compatible color groups using geometric shapes, as a determinant of COLOR CHORDS color combinations of two, three, four or more, according to The mathematical analytical relationship that confirms the validity of his system, which the researcher reached in a previous study.

Keywords

Johannes Itten, Circle Of Colors, Harmonious, Teaching, Color Science.
\end{abstract}

\section{Introduction:}

With the aim of approving the new circle as an innovative color circle that can be relied upon in the teaching of color science and chromatic harmony for art students in general, and those studying the field of design and its structural foundations in particular, in a manner consistent with their mental and cognitive abilities in light of the current information and technological revolution, and in accordance with international standards for education and arts programs to learn the range Life. Research problem: The research problem is determined by the following question: Q- How can a color circle be modified to create compatible color groups that contribute to the development of chromatic compatibility teaching? Research hypotheses: - A modification can be made in the design of the Athen color circle to allow the creation of many times the number of compatible color groups allowed. The new color groups can be taken according to the characteristics of each one as a starting point for developing the teaching of color harmony. Research methodology: The researcher followed the analytical and experimental approach through the theoretical and practical frameworks of the research. Search results: The search concluded with several results, the most important of which are as follows:

- Modifying the design of the ATN color circle allowed the creation of twice the

*Corresponding author: art_education@helwan.com 
compatible color combinations defined by its current geometric shapes.

- The new circuit enabled the researcher to add geometric shapes that added many compatible color groups in its output.

- The compatible color groups resulting from each geometric shape on the new circle have characteristics that distinguish it from others.

- The new circle and its compatible color combinations, with their distinctive characteristics, can be adopted as a starting point for developing the teaching of color harmony.

Color is one of the most important components of the artwork, and the color groups that are characterized by harmony and harmony constitute one of the aesthetic phenomena in the structural design of the work, and the theoretical study of colors has become an essential aspect of the academic preparation of the designer and plastic artist. Many scientists and researchers interested in plastic art and color science over the past ages, until the first serious theories of color in the modern era came out to us (12 AD), at the hands of the English scientist Sir Isaac Newton (1942-1727 AD), "Which provided us with the first known color system known as the Color Circle (2 - 0), and the circle that Newton created included seven colors, including indigo (9 - 12), and in the first half of the twentieth century a new color circle appeared for the poet The German Goethe (1749 - 18 AD), followed by the emergence of several other color circles at the beginning of the twentieth century for Mansell, Chevreul, Oswald Ostwald, and then Johannes Itten, the owner of the best color schemes known so far, which are directly related to the topic The current study.

The German scientist Johannes Itten (18 - 18 AD) (He is considered one of the greatest pioneers of color theories in the modern era, and he was a lover of colors, and among his famous sayings "Colors mean life to the world, without colors appearing to us as death" (10 - 2), and he held the position of member The teaching staff of the Bauhaus School of Art and Design from 1917 to $18 \mathrm{AD}$, "and perhaps he is better known to students of color science through his book: Art Of Color, and his brief version of Elements of Color" (6-19), which was published in 1961 and translated into numerous Of languages, and it contains a summary of his experiences and the results of the numerous research conducted by him in the science of color, and his definition of the characteristic Color Harmony and the system that he devised to arrive at color compositions of Color Chords, "and still theories that represent the essence of most technical schools' information about color" (2 - 12) ), And a major component of the curricula for teaching color science and its characteristics, in most international academies of art. 
While the researcher taught the color science course to his students, and dealt with the Akkan color circle, some problems related to the color compatibility property appeared in front of him, including the lack of clarity of the scientific evidence regarding the reliability of his engineering system that he relied on in reaching compatible color groups. Through a previous study, the researcher was able to verify the validity of Atten's assumption, and the existence of "a correlation between the complementary and compatible colors of a ton in terms of the stability of the proportions of its components ... and that the compatible groups defined according to a system should contain the primary colors in equal proportions" (5-15), by subjecting these groups to processes a mathematical analysis according to For numbers that represent the relative weights of their components.

In the current study, the researcher seeks to address a new problem represented in the limited total number of compatible color groups resulting from the Antun circle according to its current design, which is insufficient to meet the needs of the contemporary student and his mental abilities in light of the current cognitive revolution.

The current research falls within the scope of experimental research that is concerned with the color harmony feature, and seeks to develop compatible color groups resulting from making adjustments in the design of the Ayna color circle, to ensure the validity of the results of the new circuit's outputs, and to define the specifications and characteristics of those color groups to serve as a platform for the development of teaching Color science and chromatic harmony for art and design students, in a manner consistent with international standards for arts and education programs, lifelong learning.

\section{Research Problem:}

The researcher seeks to design a new color circle resulting from making adjustments in the design of the Johannes Aten color circle, in a way that enables him to create compatible color groups that converge in total to four times what the Atten circuit allows, and then to determine the specifications and characteristics of the new circuit outputs from the compatible color groups after verification It is correct, with the aim of taking these results as a new starting point that contributes to the development of the teaching of color science and chromatic harmony for students of plastic arts in general and the design field in particular, in a manner that suits their mental abilities and requirements in light of the current information and knowledge revolution.

\section{The research problem is determined in the following question:}

- How can a color circle be modified to create compatible color groups that contribute to developing the teaching of color harmony? 


\section{Research Methodology:}

The research follows the descriptive, analytical and experimental approach, through a set of main axes specified for both the theoretical framework and the practical framework, and the research axes are defined in three main points, under each of which there are several sub-points linked to it and explained to it, leading to the results of the research and its recommendations. The first axis: the design of the new color circle based on a modification of the design of the Johannes Atten circle.

In this topic the researcher deals with the definition of the Johannes Akn color circle and its structural design, as well as the new modified circle, its design and method of drawing it, as well as the color groups that make up each circle, in addition to the relative weights of each spectral color in each of the two circles and its structural origins of the basic colors, from Through the following points:

\section{1- The design and definition of the Johannes Aten circuit, and the new modified} circuit:

In this point the researcher will deal with the definition and design of each circuit separately, as follows:

\section{A- The definition and construction design of the Johannes Aten color circle:}

The color circle represents the closed circular sequence of colors, which is a formal system based on connecting the ends of a strip of colored spectra. Newton was the first to design this system in $\{120 \mathrm{~m}\}$, and Akon Itten "simplified his previous color systems into a circle of twelve Luna "(2-100) represents his spectral color arrangement in a simple way that is easy to study by students without difficulty and wasting time, and among his words," The study of the twelve-part color circle forms the basis for my constructivist theory of colors "(9-30).

The structural design of a second circle consists of an equilateral triangle drawn within two circles in common in one center, divided from the center of the circle into three equal parts. An area of twelve equal spaces located between the two outer circles, each of which contains one of the color spectrums representing the ATN system (5-5). To obtain the twelve parts, each of the strings that are confined between the vertices of the triangles are divided into four equal parts. Determine the distance between the two circles, passing through the specific point at the end of the first part, to the center, and through the specific point of the beginning of the fourth part, to the center

\section{B- Definition of the new color circle and its structural design:}

The new colors circle represents one of the images of the closed circular system of colors, on which all the previous color circles are based, and it is very similar to the Antun circle of colors 
in terms of its structural system, and differs from it in the details of its formal appearance around the center, as well as the number of parts of its outer perimeter The adult (24) twentyfour parts represents the order of the spectral colors of the researcher, while the number of parts of Ayna's circle in its outer circumference (12) is twelve parts representing the order of the spectral colors in him.

The structural design of the new circle consists of an equilateral triangle drawn within two circles in common in one center, and divided from the center of the circle into three equal parts like a second circle, and the triangle is surrounded by three equilateral triangles resulting from the superposition of the large triangle with another similar to it, achieving the shape of a sixpointed star, then This star is superimposed with another similar probe that has a twelvesegment star shape, and to determine the points of its vertices on the circle, a ruler is placed from the center point of the circle through the points of intersection of the small triangles with the large triangle and down to the circumference of the circle.

In order to achieve the final shape of the circle, which is to divide its outer circumference between the two circles into twenty-four parts, we divide the small strings located between the vertices of the star shape into four equal parts, so that the end of the first and the beginning of the third with the center of the circle determine the boundary of each part, thus completing the structural design of the new circle.

\section{2- The color groups in each of the Johannes Atten circle and the new circle:}

At this point, the researcher will review the color groups that each circle contains, as follows:

A- Color groups in the Aten circle of colors:

The Aten circle contains twelve spectral colors divided into three groups, namely:

1- Basic colors: they are colors that cannot be obtained from color mixing, while other colors can be obtained from mixing them: yellow, red, blue.

2- Secondary colors: They are binary composition, produced by mixing two primary colors in fixed proportions, represented by: orange, violet, and green.

3- The medium Derived Colors: It is also binary in composition, and the product of "secondary colors that mix with the primary colors and are adjacent to the primary colors in the color circle" (4-91). It is also known as "intermediate colors that are derived from two primary colors, the proportion of one of them. Twice the ratio of the other "(5-6), represented in six spectral colors: orange yellowish, orange reddish, reddish violet, bluish violet, bluish green, and yellowish green.

B- Color groups in the new color circle: 
The new color circle contains twenty-four spectral colors divided into four groups, namely:

1- Basic colors.

2- Secondary Colors.

3- The medium Derived Colors.

These three groups are identical to the constellations of a circle in terms of components and number. Twelve spectral colors are represented in the new circle from the total of its components of (24) twenty-four colors. As for the twelve spectral colors complementing the new circle, it was classified by The researcher as a fourth color group, and set a name for it, and defined it as follows:

\section{4- The side-derived colors:}

The group of colors on both sides of each color represents an intermediate derivative in the color circle, and it expresses the color resulting from mixing the intermediate color with any of the primary or secondary colors that precede or follow it on the circle, which are binary colors that can be obtained by mixing two primary colors in different proportions And this is what the researcher will deal with in detail in a later point, and the total number is twelve spectral colors defined by the following names: orange yellowish to yellow - orange yellowish to orange orange reddish to orange - reddish orange Orange reddish to red - Violet reddish to red - Violet reddish to violet - Bluish to Violet Violet - Violet Bluish to blue - green Bluish to Blue Green Bluish to green Green yellowish to green Green yellowish to yellow.

\section{3- The relative weights of each spectral color in the Johannes Atten circle, and the new circle:}

At this point, the researcher will review the relative weights of each spectral color in the two color circles, and what is meant by the relative weight is "a determination of the quantitative ratios expressing the particles that make up each spectral color ..., which can be used in mathematical analyzes that contribute to reaching relationships Certain chromatic properties such as complementarity and compatibility confirm (5 - 6), meaning that the relative weights determine the structural origins of each spectral color of the primary colors, and the ratio of each primary color, through the following points:

A- The relative weights of each spectral color in the Johannes Aten circle: By reviewing the ATN color circle, we notice the presence of a number of 1: 3 on each spectral color, distributed as follows:

-No (1) on the primary colors, as an expression of their containment of one molecule in their original composition.

-No (2) on the secondary colors, as an expression of the fact that each color contains two 
particles, each of them of a primary color, preceding or following it.

-No (3) on the intermediate-derived colors, as an expression of each color containing in its composition one molecule of the primary color that follows the secondary color next to it, and two parts of the other base color next to it.

\section{B- Relative weights of each spectral color in the new color circle:}

Reviewing the new color circle, we notice the presence of a number of 1:5 on each spectral color, distributed as follows:

-No (1) on the primary colors.

-No (2) on the secondary colors.

-No (3) for the middle-derived colors. In the same way as it is in the Ayna circle, in terms of composition and proportions of its components of the primary colors.

-No (4) on the side-derived colors, as an expression of the containment of each color in its composition on one molecule of the basic color that follows the middle derivative color next to it, and three molecules of the other base color next to it.

-No (5) on the side-derived colors, as an expression of each color containing in its composition two molecules of the primary color that follows the secondary color next to it, and three molecules of the other primary color that precedes the intermediate derivative color next to it.

The second axis: color groups compatible with the Ayna circle and the new color circle.

In this axis, the researcher deals with the concept of color compatibility, and the correlation between it and the concept of color integration, then reviews the An-Geometric system to determine the color groups that are compatible with its color circuit, how to verify the validity of its system, and the total number of compatible color groups resulting from it, as well as the resulting compatible color groups. From the use of the ATN system on the new color circle, and the additions that the researcher put on this system to reach the largest number of those groups, in addition to a presentation of confirmed mathematical analyzes, pasting his assumption of their compatibility, through the following points:

\section{1- Chromatic compatibility and its relationship to color complementarity:}

To reveal the bonds of the correlative relationship between each of the concept of color integration and the concept of color compatibility, the researcher must first define each concept separately, by presenting the views of some color scientists, and the definition of the scientist Johannes Aten with an explanation of the types of color harmonics, as follows:

\section{A- The concept of Complementary Color:}

Many color scientists have agreed on a special definition of the concept of color complementarity, which becomes the two colors complementary when mixed together, 
resulting in the middle gray "( $\left.9_{-}\right)$, and it was supported that this definition, in arranging its color circle, each two complementary colors meet on the ends of the same diameter of the circle, ... By analyzing each pair of them, we find that it is a component of the three basic colors, ... and the result of their chemical or pigment mixing is the same as the result of mixing the primary colors, i.e. grayish black "(2-1112).

To verify the validity of the assumption that the integral colors, the two complementary colors, are the primary colors and the integrity of the relative weights that the researcher determined for the components of the colors of his circle, on the color circle yellow red blue, the researcher conducted mathematical analyzes based on the relative weights of yellowish orange and bluish violet for each color, and by analyzing the components Two intermediate colors, the sum of the particles opposite on the color circle, namely: yellowish orange - bluish-violet, it becomes clear to us that they contain the primary colors, the analysis of the relative weight of the two complementary colors with equal relative weights in their structural molecules, and by repeatedly trying on the rest of the opposite color pairs on the color circle, the same result is repeated. (5-7.(

\section{B- Harmony Color concept:}

Chromatic compatibility directly controls the completeness of the system of aesthetic values of the artwork. Compatibility is defined as "a group of colors that affect the eye a pleasant and pleasant effect, and is characterized by connection and unity despite the obvious difference between them sometimes" (1 - 114). Color scientists have tried to reach scientific foundations for the concept of chromatic compatibility, and Ernst Pfeifer reached an accurate scientific definition of color compatibility, which is that "color compatibility is a harmonious union of colors that arose out of the use of the property of fusion and the convergence of colors and their visual unions that enter as mediators for reconciliation. Among them "(2 - 11), and Akane was able to confirm all the serious opinions that preceded him, as he stated" that the color group is in a state of harmony and harmony if the product of mixing its pigments together is the medium gray $(7-1112)$.

Through the previous definitions, it becomes clear to us that there are several types of color groups that are characterized by compatibility, and they are as follows: a group based on the substance of a single spectral color, a group based on adjacent colors in the circle joined by a common color, the group of simple, complex and divided integrated colors (5 -), Which will be addressed by the researcher later.

\section{C- Correlation between chromatic complementarity and chromatic harmony:}

With their in-depth studies in color science, he and many colorists were able to reach the 
correlative relationship that combines color integrals and compatibility, and perhaps the definition that compatible color groups are evidence of the extent of the integration of each group through the stability of the basic color ratios in its components, which is what the researcher relied on in the study A precedent for verifying the reliability of the outputs of the Ayna circuit from the compatible color groups, which is also based on it in the current study to verify the validity of the outputs of the new circuit from the compatible color groups.

The third axis: color groups compatible with the new color circle and its characteristics: In this axis the researcher deals with a detailed presentation of the total number of compatible color groups resulting from the use of the modified system in the new color circle, and the characteristics and specifications of each group, to be taken as a starting point for the development of color science teaching in general, and color compatibility in particular, through the following points:

\section{1- The total number of compatible groups according to the modified ATN system on the new color circle:}

At this point, the researcher performs a classification and inventory process for the total number of compatible color groups that can be deduced from the new color circle according to the ethnographic geometric system and the modifications added to it, starting from the smallest color group represented by each pair of complementary colors to the eight color groups determined by the octagonal shape choices The color circle, in order to identify the capabilities of the Akkan system, after modifying it, to create color groups compatible with the new color circle.

\section{Results:}

The research concluded with several results, the most important of which are as follows:

1- Modifying the new design of the Akin color circle, which allowed the researcher to create twice the number of compatible color groups defined by his system from geometric shapes.

2- The design of the new circuit enabled the researcher to add geometric shapes to the system that, allowed the creation of many compatible color groups in its output.

3- The new circle provided the opportunity to deduce approximately four times the total number of compatible color groups resulting from the Ayna circle, through its modified geometry system.

4- The compatible color groups resulting from each geometric shape on the new circle have characteristics that distinguish it from others.

5- The new circle and its compatible color groups with its distinctive characteristics can 
be adopted as a starting point for developing the teaching of color harmony.

\section{Recommendations:}

1- The researcher recommends the necessity of conducting an experimental study that deals with the practical application side of the results of the current study.

2- The researcher recommends the necessity of reading the old theories related to teaching art, with contemporary analytical insights that contribute to their development.

\section{References:}

1- Ismail Shawky: (Design - Its Elements and Its Basis in Plastic Art), Zahraa Al Sharq Library, Cairo, Egypt, third edition, 2009 AD.

2- Johans Athen: (Design and Form), translated and trimmed by Sabri Abdel-Ghani, Hala for Publishing and Distribution, Cairo, Egypt, first edition, 2008 AD.

3- Adel Abdel-Rahman: (Theories of Light and Colors), Dar Al-Haramain for Printing and Publishing, Cairo, Egypt, first edition, 2008 AD.

4- Ali Muhammad Abd al-Hadi and others: (The Basics of Two-Dimensional Design), Arab Society Library for Publishing and Distribution, Amman, Jordan, First Edition, 2009.

5- Muhammad Ali Nasr: (Color groups compatible with the color circle as an investigator of one of the aesthetic phenomena in design), published research, the fourth international conference of the Faculty of Art Education - Arts and Education in the Third Millennium, the third axis - the new road map for art education, Helwan University, Egypt April 2013 AD.

6- Edith Anderson Feisner: (Colour: How to use colour in Art and Design), Laurence King Publishing Ltd, London, United Kingdom, 2006.

7- Terry Stone \& Adams Mori. :( Color Design-Work Book), Published By Rock Port, Isph,USA, 2008.

8- Neta Leland : ( Confident Color ), Published By North Light Books ,Ohio, USA, 2008.

9- http : / / aphotoTeacher . com / 2008 / 09 / 13 / The - art - of - color

10- ( http://kaufmann-mercantile.com/Johannes-Itten.)

11- Salwa Hassan Mohamed Mahmoud PASHA, ADVANTAGES OF BREATHING EXERCISES ON PIANO PERFORMANCE FOR BEGINNERS, International Journal of Education and Learning Research, Vol. 1, No. 1, 2018, pp. 10-17. 